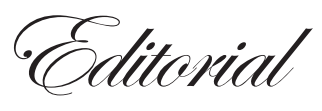

\title{
Amoebiasis and Entamoeba species: unexplored liaisons
}

\author{
Sudha Bhattacharya ${ }^{1}$, Alok Bhattacharya ${ }^{2}$
}

Schools of Environmental ${ }^{1}$ and Life $^{2}$ Sciences, Jawaharlal Nehru

University, New Delhi 110067, India

\section{Correspondence:}

Dr. Alok Bhattacharya

Email:alok.bhattacharya@gmail.com
Amoebiasis is classically defined as the disease caused by infection with Entamoeba histolytica. The human colon is also host to a number of non-pathogenic species of Entamoeba (E. coli, E. hartmanni, E. dispar and E. moshkovskii), of which the last two are morphologically indistinguishable from E. histolytica. World-wide, about 500 million people are infected with Entamoeba species, ${ }^{1}$ but the relative prevalence of each species is uncertain. About 50 million cases of invasive amoebiasis are estimated per year; with about 100000 deaths.

E. dispar is considered to be a sibling species of E. histolytica as the two are phylogenetically closest. Although it is non-pathogenic, most of the molecules considered important for amoebic pathogenesis (galactose/ $\mathrm{N}$-acetylgalactosamine-inhibitable lectin, amoeba pores, and different proteases) are found in E. dispar as well. While the search is still on for key differences in the two species, reports suggest that $E$. dispar might also be involved in tissue invasion. In a recent German study on travellers returning from the tropics, E. dispar was found in $88.3 \%$ of the Entamoeba-infected subjects (with the highest number returning from Southeast Asia and Africa). Of these, $>50 \%$ had intestinal symptoms typical of amoebiasis. ${ }^{2}$ Further, in the experimental hamster model, E. dispar was shown to cause hepatic lesions. ${ }^{3}$ These studies need to be replicated in India to re-examine the clinical status of $E$. dispar in view of its high prevalence.

$E$. histolytica and E. dispar are commonly found in the faeces of non-human primates, which is important due to its zoonotic potential. In the older literature (1908), the name $E$. nuttalli Castellani was proposed for an amoeba found in the liver abscess of a monkey (Macacus pileatus) in Colombo, Sri Lanka. Recently, E. histolytica-like amoebae have been isolated from a number of monkey species (M. mulatta, M. fascicularis and M. fuscata), mainly from captive populations. ${ }^{4,5}$ Inoculation of trophozoites into the livers of hamsters caused amoebic liver abscesses (ALAs), indicating that these monkey isolates are potentially virulent. Revival of the name $E$. nuttalli has been proposed for the monkey isolates. Importantly, from the analysis of $18 \mathrm{~S}$ rRNA sequence it appears that $E$. histolytica is closer to E. nuttalli than it is to $E$. dispar, underscoring the potential importance of this new species in human pathogenesis.

\section{Entamoeba diagnostics-historical perspective}

The earliest identification of cysts and trophozoites of Entamoeba species from stool samples was done by microscopic examination of faecal samples, which remains the method of choice in clinical settings, although this method fails to distinguish between E. histolytica and $E$. dispar. Early researchers devised several convenient culture media for xenic cultivation of the parasite. However, it was with the tireless efforts of Louis Diamond that a medium was finally designed for axenic cultivation of the parasite, ${ }^{6}$ which was a watershed event for research not only in Entamoeba but Giardia and other intestinal parasites. Xenic cultivation was 
successfully used by Sargeaunt et al. to distinguish between parasitic and non-parasitic strains by isoenzyme analysis. ${ }^{7}$ This study showed that parasites from asymptomatic individuals were distinct from those from patients with amoebiasis. On confirmation by molecular analysis, the name E. dispar was given to the non-pathogenic species, as was originally suggested by Brumpt in 1925.

The axenic cultivation method for E. histolytica made it possible to initiate biochemical and immunological studies of the parasite in the 1970s. The 1980s and 1990s saw a worldwide upsurge in molecular analysis with E. histolytica genes being cloned and sequenced by various laboratories, including those in India. The next leap in Entamoeba research came in 2005 with the availability of the genome sequence of E. histolytica strain HM-1: IMSS, which was made possible by the combined efforts of the entire international community of Entamoeba researchers. Ongoing projects are making available sequences of other E. histolytica strains and other Entamoeba species. Data from these projects have been collated into a database (AmoebaDB) which will boost research and provide us with more precise diagnostic tools and a better understanding of various pathogenic mechanisms.

\section{Entamoeba diagnostics-current scenario}

Antibody and antigen-based assays

Serological tests for the identification of E. histolytica infection are of limited value in endemic areas, but may be useful in industrialized countries, and can be helpful in the diagnosis of patients with ALA where parasite is not detected in faeces. Several commercial kits employ ELISA or indirect haemagglutination assay (IHA) for detecting anti-E. histolytica antibodies. Similarly, antigen-detection kits are also commercially manufactured. ${ }^{8}$ The antigen-based ELISA kits for E. histolytica use monoclonal antibodies against a variety of antigens such as Gal/GalNAc-specific lectin and serine-rich antigen.

\section{DNA-based diagnostic methods}

With the advent of commercial kits for purification of highquality DNA directly from stool samples, the need to culture the parasite is circumvented. PCR-based assays with faecal DNA have a greater specificity and sensitivity, and are increasingly being used both in conventional PCR and in high throughput studies. Further technological advances may make them affordable and simpler to use in clinical settings.

Most DNA-based methods use primers and probes from the small subunit (ssu) rRNA gene due to its high copy number and ubiquitous presence. The closely related $E$. histolytica and E. dispar, as well as E. moshkovskii, have been distinguished from one another using primers from appropriate regions of the ssu rRNA gene. ${ }^{9,10}$ Multiplex real-time PCR formats have been used for simultaneous detection of common intestinal parasites (Entamoeba, Giardia, Cryptosporidium) by using primers specific for each organism. Recently a singleplex, real-time PCR application called TaqMan Array Card, which works in a 380-well format, has been devised for simultaneous detection of 19 enteropathogens, including protozoa, bacteria, viruses and helminths. ${ }^{11}$

\section{Methods for genotyping E. histolytica strains}

E. histolytica infection typically results in a spectrum of clinical manifestations ranging from asymptomatic to colonic invasion and liver abscess. To what extent might this be due to the variable virulence potential of parasitic strains in our environment remains elusive. However, the necessary tools are being developed to arrive at an answer. Some of the proposed genotyping methods are based on the variable number of short tandem repeats within the genes, or linked to the tRNA genes. ${ }^{12}$ Recently a multi-locus sequence typing method has been developed to identify single nucleotide polymorphisms (SNPs) for genotyping. ${ }^{13}$ Our laboratory has developed a new approach based on changes in the genomic location of retrotransposons. ${ }^{14}$ All these studies indicate that extensive diversity exists in the clinical isolates of $E$. histolytica even from the same geographical origin. With further refinement it should be possible to identify markers or SNPs associated with virulence.

\section{Entamoeba diagnostics—studies from India}

Since India is endemic for the parasite, the familiarity with the organism is high in clinical testing laboratories. However, most of the routine diagnosis of faecal samples is done by microscopy, which cannot differentiate pathogenic from nonpathogenic species. Recently, Dr Parija's group has done the first systematic identification of Entamoeba spp. (E. histolytica, E. dispar and E. moshkovskii) from faecal samples 
by PCR-based methods. ${ }^{10}$ Importantly, they report that of all the samples that scored positive for Entamoeba by microscopy, only $34 \%$ were E. histolytica-the most prevalent being $E$. dispar. In a collaborative study with the All India Institute of Medical Sciences (AIIMS), our group showed statistically significant differences in gut bacteria in asymptomatic individuals and patients with ALA. These changes in gut flora could impact the final outcome of the disease. Two anaerobic bacterial species were detected in pus samples of ALA, and PCR revealed the presence of bacterial metronidazole-resistance genes in faecal and pus samples. ${ }^{15,16}$

\section{Future directions}

Entamoeba research in the past few decades has generated a number of valuable resources, including genome and transcriptome sequences, and technologies for gene manipulation such as stable transfection, induced gene expression and downregulation of genes. Such resources will undoubtedly help future researchers to address some of the key unanswered questions, the foremost being (i) Identification of parasitic strains from clinical samples and correlation with severity and type of disease; (ii) determination of genetic and physiological factors of the host that are correlated with invasive and extra-intestinal disease; (iii) identification of molecules and pathways involved in pathogenic processes; and (iv) combating drug resistance.

In the Indian context, the parasite continues to cause distress and morbidity to millions of people; and patients with ALA continue to need critical hospital support. Therefore, while the parasite has not left us, we seem to have left the parasite. Unfortunately, reliable epidemiological data do not exist from any part of India to help us understand the dynamic relationship between different Entamoeba species, their persistence in the human host and contribution to clinical outcome. Research efforts in amoebiasis both at the basic and clinical levels have diminished, and we are perilously close to negligible Entamoeba research. Considering how prone these parasites are to genetic diversity and population dynamics, it is prudent to maintain our research engagement with the parasite. We owe this to our patients.

\section{References}

1. WHO/PAHO/UNESCO report: A consultation with expert on amoebiasis. Mexico City, Mexico. 28-29 January, 1997. Epidemiol Bull. 1997;18:13-4.

2. Herbinger KH, Fleischmann E, Weber C, Perona P, Loscher T, Bretzel G. Epidemiological, clinical, and diagnostic data on intestinal infections with Entamoeba histolytica and Entamoeba dispar among returning travelers. Infection. 2011;39:527-35.

3. Guzman-Silva MA, Santos HL, Peralta RS, Peralta JM, de Macedo HW. Experimental amoebic liver abscess in hamsters caused by trophozoites of a Brazilian strain of Entamoeba dispar. Exp Parasitol. 2013;134:39-47.

4. Takano J, Tachibana H, Kato M, Narita T, Yanagi T, Yasutomi Y, et al. DNA characterization of simian Entamoeba histolytica-like strains to differentiate them from Entamoeba histolytica. Parasitol Res. 2009;105:929-37.

5. Tachibana H, Yanagi T, Akatsuka A, Kobayashi S, Kanbara H, Tsutsumi V. Isolation and characterization of a potentially virulent species Entamoeba nuttalli from captive Japanese macaques. Parasitology. 2009;136:1169-77.

6. Clark CG, Diamond LS. Methods for cultivation of luminal parasitic protists of clinical importance. Clin Microbiol Rev. 2002;15:329-41.

7. Sargeaunt PG, Williams JE, Grene JD. The differentiation of invasive and non-invasive Entamoeba histolytica by isoenzyme electrophoresis. Trans R Soc Trop Med Hyg. 1978;72:519-21.

8. Fotedar R, Stark D, Beebe N, Marriott D, Ellis J, Harkness J. Laboratory diagnostic techniques for Entamoeba species. Clin Microbiol Rev. 2007;20:511-32. [Table of contents]

9. Verweij JJ, Campbell BE, Gasser RB. Differentiation of Entamoeba histolytica from Entamoeba dispar by PCR-coupled nonisotopic SSCP analysis. Electrophoresis. 2006;27:4419-22.

10. Khairnar K, Parija SC. A novel nested multiplex polymerase chain reaction (PCR) assay for differential detection of Entamoeba histolytica, E. moshkovskii and E. dispar DNA in stool samples. BMC Microbiology. 2007;7:47.

11. Liu J, Gratz J, Amour C, Kibiki G, Becker S, Janaki L, et al. A laboratory-developed TaqMan Array Card for simultaneous detection of 19 enteropathogens. J Clin Microbiol. 2013;51:47280.

12. Ali IK, Zaki M, Clark CG. Use of PCR amplification of tRNA gene-linked short tandem repeats for genotyping Entamoeba histolytica. J Clin Microbiol. 2005;43:5842-7.

13. Gilchrist CA, Ali IK, Kabir M, Alam F, Scherbakova S, Ferlanti E, et al. A Multilocus Sequence Typing System (MLST) reveals a high level of diversity and a genetic component to Entamoeba histolytica virulence. BMC Microbiol. 2012;12:151.

14. Kumari V, Iyer LR, Roy R, Bhargava V, Panda S, Paul J, et al. Genomic distribution of SINEs in Entamoeba histolytica strains: Implication for genotyping. BMC Genomics. 2013: [Communicated].

15. Rani R, Murthy RS, Bhattacharya S, Ahuja V, Rizvi MA, Paul J. Changes in bacterial profile during amebiasis: demonstration of anaerobic bacteria in ALA pus samples. Am J Trop Med Hyg. 2006;75:880-5.

16. Verma AK, Verma R, Ahuja V, Paul J. Real-time analysis of gut flora in Entamoeba histolytica infected patients of Northern India. BMC Microbiol. 2012;12:183. 